\title{
Domain State Model for Exchange Bias: Influence of Structural Defects on Exchange $\mathrm{Bias}$ in $\mathrm{Co} / \mathrm{CoO}$
}

\author{
Bernd Beschoten ${ }^{1}$, Andrea Tillmanns ${ }^{1}$, Jan Keller ${ }^{1}$, Gernot Güntherodt ${ }^{1}$, \\ Ulich Nowak ${ }^{2}$, and Klaus D. Usadel ${ }^{2}$ \\ 1. Physikalisches Institut, RWTH Aachen, \\ Templergraben 55, 52056 Aachen, Germany \\ 2 Theoretische Tieftemperaturphysik, Gerhard-Mercator-Universität Duisburg, \\ 47048 Duisburg, Germany
}

\begin{abstract}
The exchange bias coupling at ferromagnetic/antiferromagnetic interfaces in epitaxially grown $\mathrm{Co} / \mathrm{CoO}$ bilayers can be intentionally enhanced by a factor of up to 4 if the antiferromagnetic CoO layer is diluted by non-magnetic defects in its volume part away from the interface. Monte Carlo simulations of a simple model consisting of a ferromagnetic layer exchange coupled to a diluted antiferromagnetic layer show exchange bias of the right order of magnitude and qualitatively reproduce the experimentally observed dependence of the exchange bias field on the number of defects. The exchange bias results from a domain state in the antiferromagnet, which is formed during field cooling and carries an irreversible domain state magnetization. Apart from intentionally introduced non-magnetic defects, also structural defects can enhance the exchange bias coupling. Twin boundaries in undiluted CoO increase the exchange bias coupling in $\mathrm{Co} / \mathrm{COO}$ by more than a factor of $2 \mathrm{com}-$ pared to untwined samples. This observation indicates that structural defects in the antiferromagnet, such as twin or grain boundaries, might also stabilize a domain state, suggesting that the domain state model for exchange bias is more generally applicable to understand the origin of the exchange bias phenomenon.
\end{abstract}

\section{Introduction}

Direct exchange coupling at the interface between a ferromagnetic (FM) and an antiferromagnetic (AFM) layer may result in exchange biasing, which induces a unidirectional anisotropy of the FM layer. This unidirectional anisotropy causes a shift of the hysteresis loop along the magnetic field axis. The magnitude of the field shift is called the exchange bias (EB) field $B_{\mathrm{EB}}$. Usually, the EB shift occurs after field cooling the system with a saturated FM layer below the Néel temperature $T_{\mathrm{N}}$ of the AFM layer or by layer deposition in an external magnetic field. Despite more than four decades of research since its discovery $[1,2]$ and the application in commercially available magnetic sensor devices $[3,4]$, the microscopic understanding of the exchange bias EB 
effect is still not fully established. For a review of the vast literature on EB the reader is referred to a recent article by Nogués and Schuller [5].

The identification of the microscopic spin structure of the AFM layer close to the interface to the FM layer is of fundamental relevance for the microscopic origin of the $\mathrm{EB}$ phenomena. Recently, we studied the $\mathrm{EB}$ in ferro-/antiferromagnetic $\mathrm{Co} / \mathrm{CoO}$ bilayers as a function of volume defects in the antiferromagnet. Of particular importance in this study was the observation that the non-magnetic defects in the volume of the AFM layer can enhance the EB by a factor of up to four $[6,7]$. The defects were not intentionally placed at the FM/AFM interface but rather throughout the volume part of the AFM layer. Therefore, the observed $\mathrm{EB}$ is primarily not due to disorder or defects at the interface. Rather, the strong dependence of the $\mathrm{EB}$ field on the dilution of the AFM layer was concluded to have its origin in the formation of a domain state in the volume of the AFM. This domain state gives rise to a small but significant excess of moments at the FM/AFM in-

terface which causes and controls EB. The 'domain state' (DS) model gives a novel microscopic description of exchange bias, which is supported by Monte Carlo (MC) simulations $[6,8]$.

The article is organized as follows. We will give an overview about some basic models for EB in section 2. In section 3 the domain state model for exchange bias is presented and the most important experimental and theoretical results are given including the domain state magnetization in section 4 . The relevance of structural defects (twin boundaries) in $\mathrm{Co} / \mathrm{CoO}$ and their link to the DS model is discussed in section 5. Finally, we conclude in the last section.

\section{Models for Exchange Bias}

One of the puzzling observations in $\mathrm{EB}$ systems is the large variation of the $\mathrm{EB}$ coupling strength even between the same type of FM/AFM material systems [5]. In order to better compare the EB coupling strength between various $F M / A F M$ systems independent of the FM material and its thickness, the $\mathrm{EB}$ coupling constant or interface energy $k_{\mathrm{EB}}$ is often used, which equals the interface energy per unit area

$$
k_{\mathrm{EB}}=B_{\mathrm{EB}} M_{\mathrm{FM}} t_{\mathrm{FM}},
$$

where $M_{\mathrm{FM}}$ and $t_{\mathrm{FM}}$ are the saturation magnetization and the thickness of the FM layer. This relation results from a simple energy argument for which the Zeeman energy of the FM in an external magnetic field $E_{\mathrm{Z}}=\mu_{0} M_{\mathrm{FM}} H$ equals the interface energy normalized to its volume $E_{\mathrm{I}}=k_{\mathrm{EB}} / t_{\mathrm{F}^{\prime} \mathrm{M}}$. Here, the AFM acts as an additional external magnetic field, which induces a shift of the magnetic hysteresis loop by $B_{\mathrm{EB}}$.

In the simplest model for $\mathrm{EB}$, the interface between the FM and the AFM layer is perfectly flat and defect free with the moments of the AFM interface 
(a)

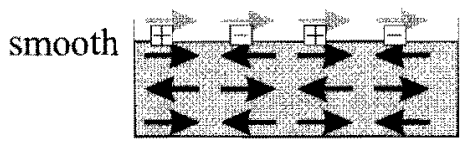

(c)

rough

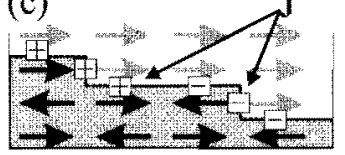

(b) uncompensated

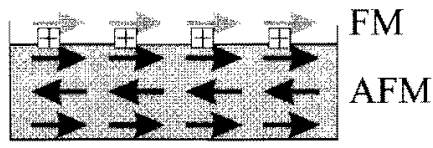

(d)

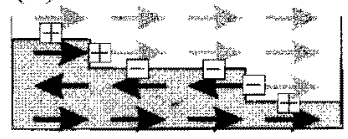

Fig. 1. Schematic structures of magnetic moments at smooth and rough (stepped) FM/AFM interfaces. The AFM interface layer is either compensated (a), (c) or uncompensated (b),(d). The plus and minus signs represent energetically favorable and unfavorable orientations of FM/AFM moment pairs. A ferromagnetic interface coupling $(+\operatorname{sign})$ is assumed

layer either being compensated or uncompensated. Additionally, the AFM layer is assumed to have an infinite anisotropy, i.e. the AFM spins are either oriented parallel or antiparallel to the FM magnetization. For a compensated moment structure (see. Fig. 1(a)), the exchange couplings between neighboring FM and AFM moments are energetically favorable ( + sign for FM interface coupling $J$ ) and unfavorable $(-\operatorname{sign})$ in alternating order. Therefore the macroscopic interface exchange coupling energy vanishes and no EB exists. However, for an uncompensated AFM moment surface, all FM/AFM moment pairs are either favorable (Fig. 1(b)) or unfavorable, which yields different interface exchange coupling energies with $k_{\mathrm{EB}}=\left(N J_{\mathrm{INT}}\right) / F$, where $N / F$ is the number of moment pairs per unit area and $J_{\mathrm{INT}}$ is the coupling energy of a FM/AFM moment pair. The resulting EB field is

$$
B_{\mathrm{EB}}=\frac{N J_{\mathrm{INT}}}{F} \frac{1}{M_{\mathrm{FM}} \cdot t_{\mathrm{FM}}} .
$$

Using reasonable numbers, this equation gives EB fields which are typically two orders of magnitude larger than the experimentally observed values. However, any realistic experimental system has a certain interface roughness. If statistically distributed, these, i.e., stepped interfaces lead to zero interface exchange coupling energy and thus zero EB coupling for both compensated (Fig. 1(c)) and uncompensated (Fig. 1(d)) AFM moment interface layers.

Malozemoff was the first one pointing out the role of AFM domains for the $\mathrm{EB}$ effect due to interface roughness $[10,11,12]$. These domains, which were assumed perpendicular to the FM/AFM interface (see Fig. 2), were supposed to occur during cooling the system below $T_{N}$ in the presence of the magnetized FM layer and to carry a small net magnetization at the FM/AFM interface. This magnetization was thought to be increasingly stabilized towards low temperatures, consequently shifting the hysteresis loop. 


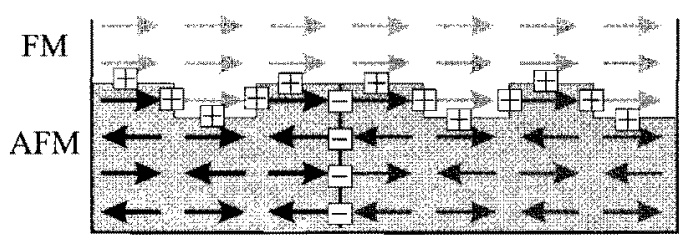

Fig. 2. Schematic structure of magnetic moments at FM/AFM interface, A domain wall in the AFM layer perpendicular to the interface leads to a net interface coupling (plus signs)

However, the formation of domain walls caused only by interface roughness is energetically unfavorable and has not directly been proven.

The magnetic linear dichroism effect and photoelectron emission spectroscopy has recently been used to probe and to image both FM and AFM domains in FM/AFM exchange coupled systems $[13,9]$. These domains have been shown to be coinciding and to provide evidence of EB coupling on a local scale.

In the micro magnetic model by Schulthess and Butler $[15,16] \mathrm{EB}$ is only obtained if uncompensated frozen in moments are assumed at the interface. However their origin and stability during magnetization reversal is not explained within this model, although uncompensated moments have been observed experimentally [17]. Other EB models $[18,19]$ assume the formation of a domain wall in the AFM parallel to the FM/AFM interface.

\section{Domain State Model for Exchange Bias}

The domain state (DS) model for exchange bias provides a microscopic description for the existence and stabilization of volume domains within the AFM layer carrying a surplus magnetization which causes and controls the EB coupling at the FM/AFM interface [6], which goes beyond the approach by Malozemoff. In the DS model, the stabilization of AFM domains is not due to interfacial roughness, but rather induced and stabilized by the existence of volume defects in the AFM layer. The DS model links the physics of Diluted AntiFerromagnets in an external Field (DAFF) 20,21$]$ to the coupling mechanism of exchange coupled FM/AFM layers.

It is well known that a three-dimensional DAFF develops a domain state when cooled below its Néel temperature $[20,21]$. The driving force for the domain formation is a statistical imbalance of the number of impurities of the two sub-lattices in a finite region of the DAFF. This leads to a net magnetization which couples to the external field. The necessary energy increase due to domain wall formation can be minimized if the domain walls preferentially pass the non-magnetic defects at no cost of exchange energy $[22,23]$. Hence, defects substantially favor the formation of domains in the DAFF.

This is illustrated in Fig. 3, where the black dots represent non-magnetic atoms or defects. The dotted line separates a domain inside with a staggered 


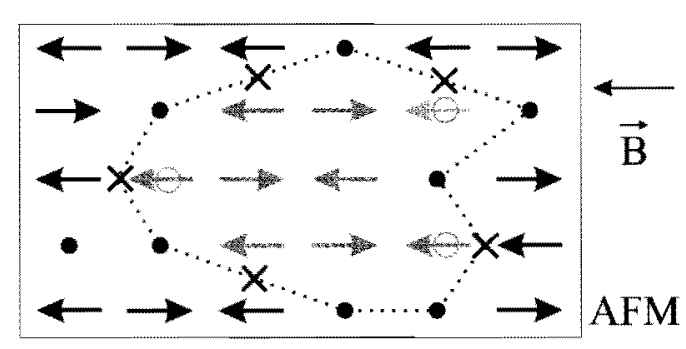

Fig. 3. Schematic structure of magnetic moments in a diluted antiferromagnet. The domain wall (dotted line) preferentially goes through defects (black dots). Frustrated bonds $(x)$ and uncompensated moments (O) are labeled along the domain wall

magnetization reversed compared to the staggered magnetization outside the domain. There are 3 uncompensated moments $(O)$ inside the domain, while 5 frustrated bonds exist along the domain boundary $(x)$. This spin configuration can be stabilized for a cooling field $B>(5 / 3)\left|J_{\mathrm{AFM}}\right|$.

As a result, the DAFF will form a domain state when cooled from its paramagnetic state in an external magnetic field. This domain state will carry a certain surplus magnetization compared to the AFM state. The magnetization of the domain can be determined by field heating from an initially ordered state, which is prepared after first zero-field cooling the system. The difference of the field cooled and the field heated magnetizations corresponds to the irreversible domains state (IDS) magnetization $m_{10 S}$ of the DAFF [8].

In the DS model for EB, the IDS magnetization of diluted AFM layers, which directly controls the EB coupling at the FM/AFM interface, originates from the AFM interface layer. The EB coupling can be established after cooling the system below $T_{\mathrm{N}}$ of the AFM layer either in an external magnetic field or with the FM layer remanently magnetized, which acts as an effective interface exchange field. Therefore, the diluted AFM can be expected to form a DS carrying IDS magnetization similar to that of a DAFF after field cooling.

The DS model is based on a combined experimental and theoretical effort. Experimentally, we studied the exchange bias in FM/AFM (111)-oriented Co/CoO bilayers grown by molecular beam epitaxy on (0001)-oriented sapphire substrates as a function of volume defects in the AFM [6,7]. The nonmagnetic defects (dilution) for $\mathrm{Co}$ in $\mathrm{CoO}$ were realized in two ways: (i) by over oxidizing $\mathrm{CoO}$, leading to $\mathrm{Co}$ deficiencies in $\mathrm{Co}_{1-y} \mathrm{O}$ and (ii) by substituting non-magnetic $\mathrm{Mg}$ ions for magnetic $\mathrm{Co}$ in $\mathrm{Co}_{1-x} \mathrm{Mg}_{x} \mathrm{O}$. For all samples investigated a $0.4 \mathrm{~nm}$ thick $\mathrm{COO}$ layer with minimum defect concentration was placed at the interface. Therefore, in these systems the observed EB is primarily not due to disorder or defects at the interface.

Theoretically, MC simulations were performed at finite temperatures of a model consisting of a FM monolayer exchange coupled to a diluted AFM film consisting of 9 layers. The FM layer is described by a classical Heisenberg model. The dipolar interaction is approximated by including an anisotropy term leading to an in-plane magnetization. Also, an easy axis in the FM 
(anisotropy constant $0.1 J_{\mathrm{FM}}$ ) was introduced in order to obtain well-defined hysteresis loops. In view of the rather strong anisotropy in $\mathrm{CoO}$ an Ising Hamiltonian was assumed for the DAFF with the easy axis is parallel to that of the FM. For the coupling between AFM and FM the same coupling constant $\left(J_{\mathrm{INT}}=J_{\mathrm{AFM}}\right)$ as for the AFM. In order to model the same interface structure for all simulations the interface monolayer of the AFM was fixed at a dilution of $50 \%$, while the dilution in the volume of the AFM film $(8$ layers) was varied, in analogy to what was done in the experiment.

The most striking experimental and theoretical results are summarized in Fig. 4. In Fig. 4(a) the almost unbiased hysteresis loop of $\mathrm{Co} / \mathrm{CoO}\left(p\left(\mathrm{O}_{2}\right)\right.$ $\left.=3.3 \times 10^{-7} \mathrm{mbar}\right)$ is shown with the biased loop of $\mathrm{Co} / \mathrm{CO}_{1-y} \mathrm{O}\left(p\left(\mathrm{O}_{2}\right)=\right.$ $5 \times 10^{-6} \mathrm{mbar}$ ) at $T=20 \mathrm{~K}$. The dilution dependence of $\left|B_{\mathrm{EB}}\right|$ for both $\mathrm{Mg}$ diluted $\mathrm{Co} / \mathrm{Co}_{1-x} \mathrm{Mg}_{x} \mathrm{O}$ and $\mathrm{Co}$ deficient $\mathrm{Co} / \mathrm{Co}_{1-y} \mathrm{O}$ samples are shown in Figs. 4(b) and (c), respectively. For comparison, Fig. 4(d) shows the EB as a function of dilution of the AFM volume as obtained from MC simulations.

(a)

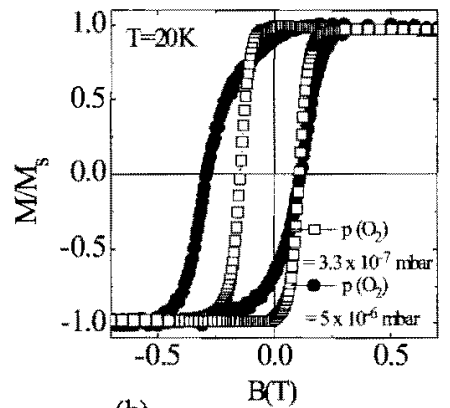

(b)

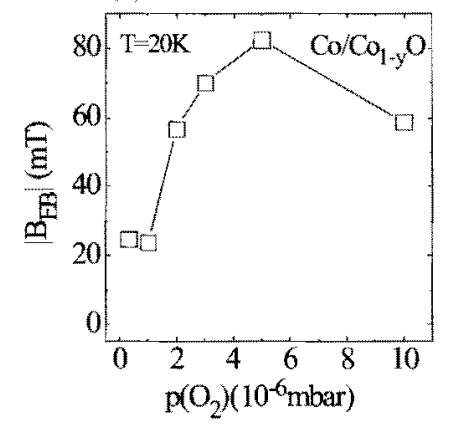

(c)
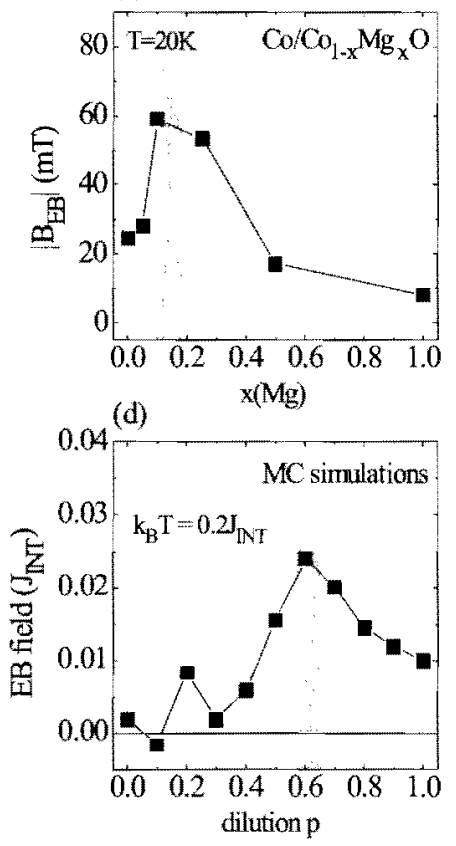

Fig. 4. (a) Hysteresis loops of $\mathrm{Co}_{-y} \mathrm{O} / \mathrm{Co} / \mathrm{Al}_{2} \mathrm{O}_{3}$ (0001) at $T=20 \mathrm{~K}$ with the Cor-y $\mathrm{O}$ prepared at $p\left(\mathrm{O}_{2}\right)=3 \times 10^{-7} \mathrm{mbar}$ and $p\left(\mathrm{O}_{2}\right)=5 \times 10^{-6} \mathrm{mbar}$, respectively. (b) EB field vs. Mg concentration in $\mathrm{Co}_{1-x} \mathrm{Mg}_{x} \mathrm{O}$ and (c) vs. oxygen pressure during deposition of $\mathrm{CO}_{1-y} \mathrm{O}$ at $20 \mathrm{~K}$. (d) Simulated $\mathrm{EB}$ as a function of dilution $p$ of the AFM layer for $k_{\mathrm{B}} T=0.2 J_{\mathrm{INT}}$ 
The experimental results were obtained after field cooling the samples from above $T_{N}$ in a magnetic field of $B_{\mathrm{FC}}=5 \mathrm{~T}$ oriented parallel to the plane of the film. EB values were then determined from hysteresis loops measured at $T=20 \mathrm{~K}$ by SQUID magnetometry. As is seen in Fig. 4(b) and (c) the EB can be enhanced by a factor of 3 to 4 for both types of defects in the AFM layer. Maximum enhancement is obtained for $x(\mathrm{Mg})=0.1$ and $p\left(\mathrm{O}_{2}\right)=5 \times 10^{-6} \mathrm{mbar}$. These sample are optimally diluted. Samples with the lowest defect concentration were prepared for $x(\mathrm{Mg})=0.0$ and an oxygen pressure of $p\left(\mathrm{O}_{2}\right)=3.3 \times 10^{-7} \mathrm{mbar}$. As the $\mathrm{CoO}$ layer of the latter samples still shows structural defects (see section 5.2), such as twin boundaries, these samples are referred to as unintentionally diluted. Typical hysteresis loops of both unintentionally and optimally diluted $\mathrm{Co} / \mathrm{Co}_{1-y} \mathrm{O}$ samples are shown in Fig. 4(a). Apart from an enhancement of EB, the defects also increase the coercive fields and significantly broaden the transition width of magnetization reversal.

The dilution dependence of the $\mathrm{EB}$ as extracted from $\mathrm{MC}$ simulations is shown in Fig. 4(d). The overall qualitative agreement with the experimental findings is clearly given. Since dilution favors the formation of domains it leads to an increase of the magnetization in the AFM and thus to a strong increase of the EB upon dilution. The AFM magnetization can directly be measured as will be shown in the next section. For larger dilutions the EB drops again due to a loss of connectivity in the AFM spin lattice.

For small dilutions, the DS model only shows a very small EB (Fig. 4(d)), although the unintentionally diluted samples still have a rather large finite $\mathrm{EB}$ (Fig. 4(b),(c)). In addition, the concentration for optimally diluted samples significantly differs between experiment $(x(\mathrm{Mg}) \approx 0.15)$ and theory $(p \approx 0.6)$. This difference might be explained by the presence of twins, grains and grain boundaries which are present in the $\mathrm{CoO} / \mathrm{Co} / \mathrm{Al}_{2} \mathrm{O}_{3}$ samples or by the existence of other structural defects in the AFM layer. Such defects are presently not included in the DS model, but they are also expected to favor domain wall formation similar to the intentionally introduced defects, thus leading to a finite $\mathrm{EB}$ for unintentionally diluted samples.

In section 5 we directly demonstrate that twin boundaries in the AFM $\mathrm{CoO}$ enhance the $\mathrm{EB}$ with respect to untwined samples.

\section{Domain State Magnetization}

Like most conventional magnetization probes, SQUID magnetometry is not layer or element specific but rather measures the whole FM/AFM bilayer magnetization. In addition to the magnetization of the FM layer, both interface and volume magnetization of the diluted AFM layer will therefore contribute to the total magnetization. The IDS magnetization of the AFM might be identified by a vertical shift of the total hysteresis loop. This is schematically illustrated in Fig. 5(a). A surplus magnetization of the AFM 

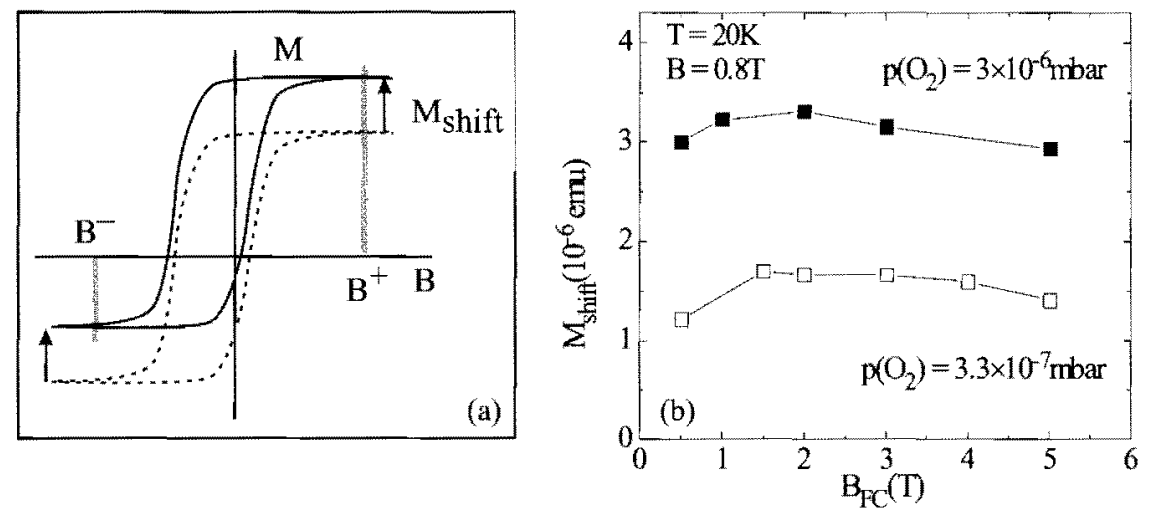

Fig. 5. (a) Schematic illustration of vertical magnetization shift due to irreversible uncompensated magnetic moments or the IDS magnetization of the AFM interface layer.(b) Vertical magnetization shift $M_{\text {shift }}$ vs. cooling field for $\mathrm{Co} / \mathrm{CO}_{1-y} \mathrm{O}$ samples with $\mathrm{Co}_{-y} \mathrm{O}$ prepared at different oxygen partial pressures. Data are taken at $B= \pm 0.8 \mathrm{~T}$ and $T=20 \mathrm{~K}$ and are extracted as described in the text

layer in an exchange coupled FM/AFM bilayer system was first identified in hysteresis loops of $\mathrm{Fe} / \mathrm{FeF}_{2}$ and $\mathrm{Fe} / \mathrm{MnF}_{2}$ [24]. Both, positive and negative vertical shifts were found and attributed to positive (ferromagnetic) and negative (antiferromagnetic) FM/AFM interface coupling, respectively. However, the origin of the induced uncompensated moments could not directly be assigned to either interface or volume magnetization.

In the DS model, the IDS magnetization of the AFM domain state determines the EB coupling strength. To investigate the change of the AFM magnetization with the number of introduced volume defects in the AFM layer, we performed high accuracy magnetization measurements of the vertical magnetization shift for both, the unintentionally diluted and oxygen diluted samples grown at $p\left(\mathrm{O}_{2}\right)=3.3 \times 10^{-7}$ mbar and $p\left(\mathrm{O}_{2}\right)=3 \times 10^{-6} \mathrm{mbar}$, respectively. The shift was determined at $T=20 \mathrm{~K}$ and $B= \pm 0.8 \mathrm{~T}$ with the FM layer fully saturated and is given by $M_{\text {shift }}=\left|M\left(B_{+}\right)\right|-\left|M\left(B_{-}\right)\right|$. As is seen in Fig. 5, at cooling fields larger than the saturation field of the FM layer $M_{\text {shift }}$ is positive and increases with dilution of the AFM layer at all cooling fields. This increase can directly be linked to the creation of additional volume defects in the AFM layer. It further supports the formation of domains in the AFM carrying surplus magnetization after field cooling. It is important to note that in our experiments we measure the total AFM surplus magnetization, which is not equal to the IDS magnetization. The latter originates from the AFM interface layer. However, we find striking qualitative agreement that the EB field indeed is proportional to the AFM surplus magnetization. 


\section{Role of Twin Boundaries for Exchange Bias in $\mathrm{Co} / \mathrm{CoO}$}

In this section we will investigate the relevance of twin boundaries for the EB coupling in Co/CoO layers grown on (111) oriented MgO substrates.

\subsection{Sample Preparation}

All samples discussed in Fig. 4 were grown on (0001) oriented sapphire substrates with a stacking order $\mathrm{CoO} / \mathrm{Co} / \mathrm{Al}_{2} \mathrm{O}_{3}$. Both, Co and $\mathrm{CoO}$ grow epitaxially in (111) orientation with $60^{\circ}$ twins in the $\mathrm{CoO}$ layer [6] (see also section 5.2). In order to grow epitaxial but untwined $\mathrm{CoO}$, we chose (111) oriented $\mathrm{MgO}$ as a new substrate. $\mathrm{MgO}$ is iso structural to $\mathrm{CoO}$ and has a moderate lattice mismatch of $1.4 \%$. Two sets of samples with different stacking orders were prepared: (I) $\mathrm{CoO} / \mathrm{Co} / \mathrm{MgO}$ and (II) $\mathrm{Pt}-\mathrm{cap} / \mathrm{Co} / \mathrm{CoO} / \mathrm{MgO}$. As the EB coupling is well known to depend on both FM and AFM layer thickness, we chose independent of the stacking sequence the same layer thicknesses identical to those previously chosen for the growth on sapphire $6 \mathrm{~nm}$ for $\mathrm{Co}$ and $20 \mathrm{~nm}$ for $\mathrm{CoO}$ ). This enables a reliable comparison of the $\mathrm{EB}$ coupling between the different samples.

For all samples the oxygen pressure was $p\left(\mathrm{O}_{2}\right)=3.3 \times 10^{-7} \mathrm{mbar}$ during the growth of $\mathrm{CoO}$. As it was discussed in Fig. 4 this should lead to unintentionally diluted samples for all cases. Further details of the growth conditions are described elsewhere [6].

\subsection{Structural Properties}

All samples were characterized in situ by reflection high energy electron diffraction (RHEED). First we will focus on the results for the samples grown on the sapphire substrates. RHEED images of the $20 \mathrm{~nm}$ thick $\mathrm{Co}_{1-y} \mathrm{O}$ layers are shown in Fig. 6 grown at an oxygen pressure of $p\left(\mathrm{O}_{2}\right)=3.3 \times 10^{-7}$ mbar (upper panel) and $p\left(\mathrm{O}_{2}\right)=5 \times 10^{-6} \mathrm{mbar}$ (lower panel), respectively. Fig. $6(a)$ and (e) show diffraction patterns for the electron beam incident parallel to the [1120]-direction $\left(0^{\circ}\right)$ of the $(0001)$ oriented sapphire substrate, while in Fig. 6(b) and (f) the beam is parallel to the [1010]-direction $\left(30^{\circ}\right)$.

All RHEED patterns of the CoO layer show transmission images, i. e. diffraction from three-dimensional structures. In order to explain the observed RHEED patterns a (111) orientation of fec $\mathrm{Co}_{1-y} \mathrm{O}$ is assumed. The calculated diffraction patterns are shown on the right panels in Fig. 6. The full circles represent reciprocal lattice points that fulfill the diffraction condition for an fcc lattice. The pattern of these points does not reproduce the double spot structures as observed for all oxygen concentrations in the $30^{\circ}$ direction. In order to explain these diffraction patterns, we furthermore have 


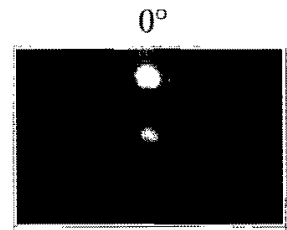

(a)

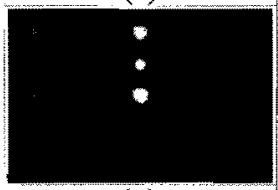

(e)

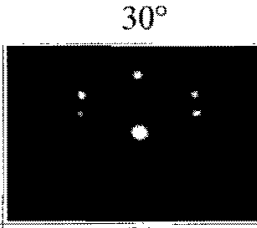

(b)

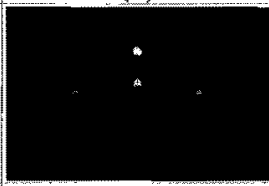

(f)

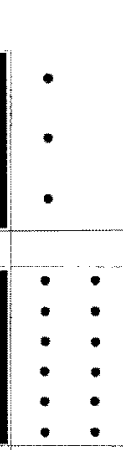

(g)

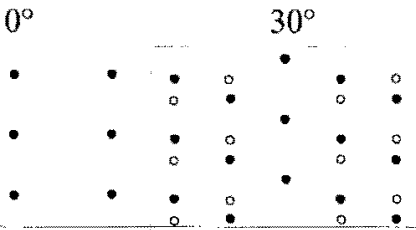

(d)
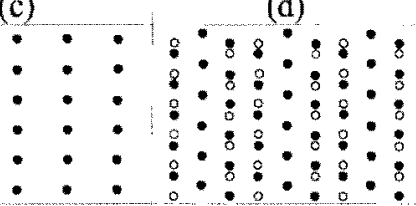

(h)

Fig. 6. RHEED images of two $20 \mathrm{~nm} \mathrm{Co}_{1-3} \mathrm{O}$ layers prepared at $p\left(\mathrm{O}_{2}\right)=$ $3.3 \times 10^{-7}$ mbar (upper panel (a),(b)) and at $p\left(\mathrm{O}_{2}\right)=5 \times 10^{-6}$ mbar (lower panel (e),(f)). (c), (d) and (g), (h) show simulated diffraction patterns of $\mathrm{CO}_{1-y} \mathrm{O}$ (111) with $60^{\circ}$ in-plane twins. The vertical panels show the patterns for $0^{\circ}$ and $30^{\circ}$ in plane orientation of the incident electron beam relative to the sapphire [1120] axis

to assume that $\mathrm{CO}_{1-y} \mathrm{O}$ grows in a twined structure where crystallites are oriented $60^{\circ}$ relative to each other. The additional spots for the twined structure are included in the calculated pattern by open circles.

The intentionally introduced defects in the CoO layer can structurally directly be identified by the appearance of additional diffraction spots in the RHEED images of diluted AFM layers (Fig. 6(f)). A more detailed analysis about these defects is given in Refs. [6,7].

We now discuss the RHEED patterns of $\mathrm{Co}_{1-y} \mathrm{O}$ layers prepared on $\mathrm{MgO}$ (111), which are shown in Fig. 7 for type I samples $(\mathrm{CoO} / \mathrm{Co} / \mathrm{MgO})$ in upper panel and type II samples $(\mathrm{Co} / \mathrm{CoO} / \mathrm{MgO})$ in the lower panel. The RHEED pattern of type I samples (Fig. 7(a),(b)) are almost identical to the RHEED pattern for the CoO grown on sapphire (see Fig. 6(a),(b)). Therefore we conclude that $\mathrm{CoO}$ grows in $(111)$ orientation with $60^{\circ}$ twins.

However, if the $\mathrm{CoO}$ layer is directly grown on $\mathrm{MgO}$ such as for type II samples, we obtain diffraction patterns as expected for an fcc (111) oriented surface without twinning (Fig. 7(e),(f)). The RHEED patterns clearly show that both types of samples are unintentionally diluted. We now have a tool to separately control structural defects such as twins and intentionally introduced dilutions (non-magnetic defects) in the AFM layer and can study their relevance for the EB coupling in exchange coupled FM/AFM bilayers.

\subsection{Magnetic Properties}

To demonstrate the influence of the twinning on the EB coupling, we show in Fig. 8 the temperature dependence of the $\mathrm{EB}$ field $\left|B_{E B}\right|$ for all undiluted samples as described in the last section. For comparison we include results for 


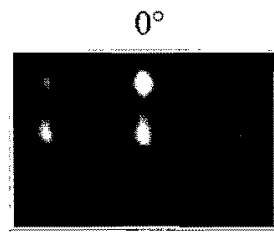

(a)

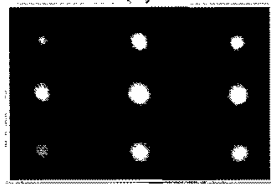

(e)

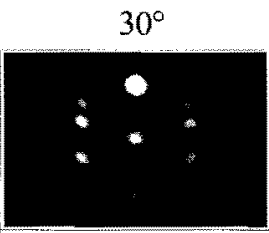

(b)

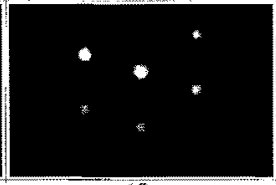

(f)

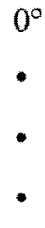

(c)

$(\mathrm{g})$

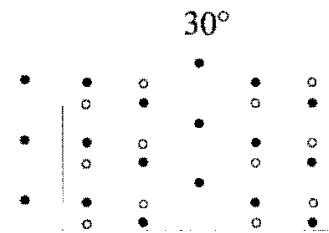

(d)

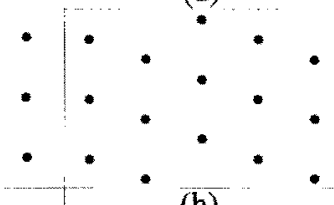

(h)

Fig. 7. RHEED images of unintentionally diluted $\mathrm{COO}$ prepared at $p\left(\mathrm{O}_{2}\right)=$ $3.3 \times 10^{-7}$ mbar grown in the stacking sequence $\mathrm{CoO} / \mathrm{Co} / \mathrm{MgO}$ (111) (upper panel, (a),(b)) and $\mathrm{Co} / \mathrm{CoO} / \mathrm{MgO}$ (111) (lower panel, (e),(f)). (c), (d) and (g), (h) show calculated diffraction patterns for twined and untwined $\mathrm{CoO}$, respectively. The vertical panels show the patterns for $0^{\circ}$ and $30^{\circ}$ in plane orientation of the incident electron beam relative to the $\mathrm{MgO}[111]$ axis

an optimally diluted $\mathrm{Co} / \mathrm{Co}_{-y} \mathrm{O}$ sample grown on sapphire (see also Fig. 4). Independent of the particular substrate chosen we find that undiluted and twined $\mathrm{Co} / \mathrm{Co}_{1-y} \mathrm{O}$ samples (open and filled squares) show almost identical magnitude and temperature dependence of the EB field. However, the undiluted and untwined sample has the smallest EB of all samples investigated, which is reduced by a factor of more than 2 compared to the twined samples.

These findings strongly suggest that twined AFM layers can also reduce the domain wall energy thus leading to domain wall formation in the volume of the AFM layer and thus to EB even without dilution.

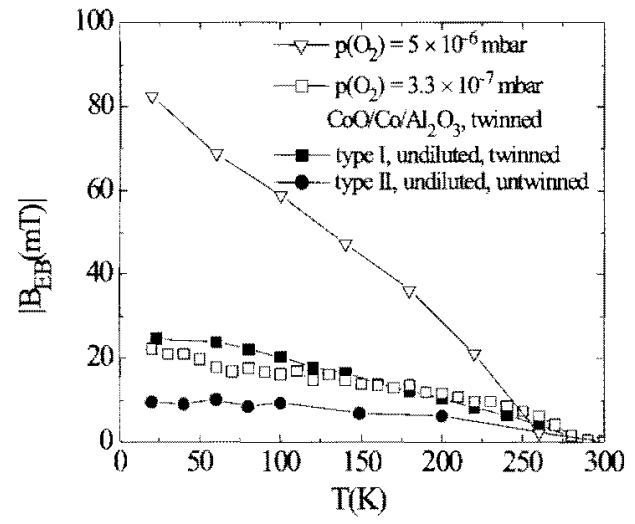

Fig. 8. Temperature dependence of $\mathrm{EB}$ field of $\mathrm{Co} / \mathrm{Co}_{1-u} \mathrm{O}$ samples grown on $\mathrm{Al}_{2} \mathrm{O}_{3}(0001)$ and $\mathrm{MgO}$ (111) substrates. Details about the samples are given in the legend and are described in the text. Note that the unintentionally diluted and untwined sample grown on $\mathrm{MgO}$ shows the smallest $\mathrm{EB}$ of all samples 


\section{Conclusions}

In conclusion, we have shown both theoretically and experimentally that the DS model for EB which is inherently based on disorder in the volume of the AFM layer gives a new and consistent insight into the microscopic mechanism of the EB effect. We could demonstrate how domains in the AFM, which were speculated about previously $[10,11,12]$, can indeed be stabilized. Moreover, we could also identify the origin of the uncompensated moments in the AFM, observed previously [17] and postulated in a previous model $[15,16]$. We have shown that the EB coupling crucially depends on the density of volume defects (such as cation deficiencies) as well as structural defects (such as grain boundaries, twin boundaries and others) in the AFM layer. This results in different spin configurations and might lead to different IDS magnetization in the AFM interface layer, which can yield a strong variation in the $\mathrm{EB}$ coupling strength.

This work has been supported by the DFG through SFB 341 and 491.

\section{References}

1. W.H. Meiklejohn and C.P. Bean: Phys. Rev. 102, 1413 (1956).

2. W.H. Meiklejohn and C.P. Bean: Phys. Rev. 105, 904 (1957).

3. B. Dieny et al.: Phys. Rev. B 43, 1297 (1991).

4. C. Tsang: J. Appl. Phys. 55, 2226 (1984).

5. J. Nogués and Ivan K. Schuller: J. Magn. Magn. Mat. 192, 203 (1999).

6. P. Miltényi, M. Gierlings, J. Keller, B. Beschoten, G. Güntherodt, U. Nowak, K.D. Usadel: Phys. Rev. Lett, 84, 4224 (2000).

7. J. Keller, F. Miltényi, B. Beschoten, G. Güntherodt, U. Nowak, and K.D. Usadel: submitted (2002).

8. U. Nowak, K.D. Usadel, J. Keller, P. Miltényi, B. Beschoten, G. Güntherodt: submitted (2002).

9. H. Ohldag, A. Scholl, F. Nolting, S. Anders, F. U. Hillebrecht, and J. Stör, Phys. Rev. Lett. 86, 2878 (2001).

10. A.P. Malozemoff: Phys. Rev. B 35, 3679 (1987).

11. A.P. Malozemoff: J. Appl. Phys. 63, 3874 (1988),

12. A.P. Malozemoff: Phys. Rev. B 37, 7673 (1988).

13. F. Nolting, A. Scholl, J. Stöhr, J.W. Seo, J. Fompeyrine, H. Siegwart, J.-P. Locquet, S. Anders, J. Lüning, E.E. Fullerton, M.F. Toney, M.R. Scheinfein and Padmore, Nature 405, 767 (2000).

14. H. Ohldag, A. Scholl, F. Nolting, S. Anders, F. U. Hillebrecht, and J. Stöhr, Phys. Rev. Lett. 86, 2878 (2001).

15. T.C. Schulthess and W.H. Butler: Phys. Rev. Lett. 81, 4516 (1998).

16. T.C. Schulthess and W.H. Butler: J. Appl. Phys. 85, 5510 (1999).

17. K. Takano, R.H. Kodama, A.E. Berkowitz, W. Cao, and G. Thomas: Phys. Rev. Lett. 79, 1130 (1997).

18. D. Mauri, H.C. Siegmann, P.S. Bagus, and E. Kay: J. Appl. Phys. 62, 3047 (1987).

19. M.D. Stiles and R.D. McMichael: Phys. Rev. B 59, 3722 (1999). 
20. W. Kleemann: Int. J. Mod. Phys. B 7, 2469 (1993).

21. D.P. Belanger: in Spin Glasses and Random Ficlds, A.P. Young (Ed.) (World Scientific, Singapore 1998).

22. U. Nowak and K. D. Usadel: Phys. Rev. B 46, 8329 (1992).

23. J. Esser, U. Nowak, and K. D. Usadel: Phys, Rev. B 55, 5866 (1997).

24. J. Nogues, C. Leighton, and I.K. Schuller: Phys. Rev. B 61, 1315 (2000). 